\title{
Nonlinear Analysis of a Quasi-Zero Stiffness Air Suspension Based on the Cell-Mapping Method
}

\author{
Long Chen, Jun Wang, Xing Xu, Xinwei Jiang and Feng Wang \\ Automotive Engineering Research Institute, University of Jiangsu, Zhenjiang, China. \\ E-mail: 1814409917@qq.com.
}

(Received 6 December 2020; accepted 9 April 2021)

\begin{abstract}
The quasi-zero stiffness system has the characteristics of low dynamic stiffness and high static stiffness, which can bring a better driving experience and lower road dynamic load at high speed on irregular roads. This paper studies a type of interconnected quasi-zero stiffness air suspension system, which has two states, namely, the noninterconnected quasi-zero stiffness air suspension and the interconnected quasi-zero stiffness air suspension, to meet the performance requirements under different loads and vehicle speed. First, the mathematical model of the nonlinear system is established based on the basic principles of fluid mechanics and thermodynamics. Then, the stability of the equilibrium point is analyzed using the Lyapunov first method, where the quantitative analysis of the attractive region of the system is conducted through the bifurcation diagram and phase diagram. By using the Taylor series expansion, cell-mapping theory and domain map of attraction, the attractive region of the system is quantitatively analyzed to obtain the parametric feasible domain under stable conditions. Finally, the performance of the quasi-zero stiffness suspension system with the selected parameters under the stability constraint is verified by simulation analysis and experiment. The results show that the system represented in this paper provides higher suspension comfort and stability.
\end{abstract}

\section{INTRODUCTION}

The low-frequency vibration of $0.5 \sim 5 \mathrm{~Hz}$ can reduce vehicle comfort and affect passenger health. ${ }^{1,2}$ Therefore, automobile designers have begun to focus on improving the performance of springs and the reliability of the control system for driving comfort and stability. The traditional coil springs and passive dampers cannot achieve the higher performance requirement of the low-frequency vibration isolation. ${ }^{2,3}$ Therefore, in the past few years, new suspension systems have steadily been developed to meet customers' higher requirement for vehicle comfort, along with maintaining high safety requirements while improving performance and reducing suspension costs, such as the teardrop-shaped spring ${ }^{4}$ and an air spring with an auxiliary chamber. ${ }^{3}$ However, these designs have the disadvantage of small load capacity or large size. ${ }^{3-5}$

The negative stiffness isolator with the negative stiffness structure in parallel with the positive stiffness structure has the useful characteristics of low dynamic stiffness and high static stiffness, which effectively reduces the resonance frequency and increases the vibration isolation range. ${ }^{6}$ Takeshi Mizuno used active control technology to realize an analytic control method of negative stiffness with linear actuators which could achieve high stiffness against the direct disturbance of the isolation. ${ }^{7}$ Daolin $\mathrm{Xu}$ developed and tested the quasi-zero stiffness system experimental prototype based on the harmonic balance method. Through a series of experimental studies, the tuning technique which adapts to load change and adjusts quasi-zero stiffness performance in the practical application was discussed. ${ }^{8}$ Zhifeng Hao and Qingjie Cao used a numerical simulation to study a quasi-zero stiffness system which revealed the complex nonlinear characteristic of the system. ${ }^{9}$ Gianluca Gatti presented a $k$-shaped biomimetic nonlin- ear spring and verified the superior energy storage and release performance of the negative stiffness structure. The stability of the four-spring structure under large deflection was also studied. ${ }^{10,12}$ An optimized quasi-zero stiffness system with a cam roller mechanism structure was proposed by Kan Ye to adapt to different loads. ${ }^{11} \mathrm{Tu}$, L. et al., applied a magnetic spring with negative stiffness to an automobile seat suspension to achieve vibration reduction. The resonance frequency vibration during the vertical excitation was reduced without affecting the bearing capacity, and excellent low-frequency suppression performance was obtained (the acceleration was reduced by $28.8 \%$ under the random excitation). ${ }^{13}$ A translational-rotational coupling quasi-zero stiffness isolator with cam-roller mechanism has also been proposed by Kan Ye to provide high-static-lowdynamic stiffness simultaneously in the translational and rotational direction. ${ }^{14}$

Above all, most negative stiffness systems have nonadjustable loads and less consideration is given to the multiattractors and bifurcation behaviour of quasi-zero stiffness nonlinear systems which are not suitable for vehicle suspension. Also, Palomares, E. et al., proposed a negative stiffness system (i.e. quasi-zero stiffness system) that was constructed by coupled air actuators and an air spring and then compared it to the passive system by experimental test and simulation which showed a $58 \%$ reduction in the resonance frequency. ${ }^{15}$ Based on this idea, a kind of quasi-zero stiffness air suspension which can connect air actuators and an air spring has been designed and the stability and parameter constraints under the stability is analysed. This paper will consider the effects of the interconnected state on the vehicle suspension which has not been studied systematically in previous studies. The result of the analysis will be verified by simulation and experiment 


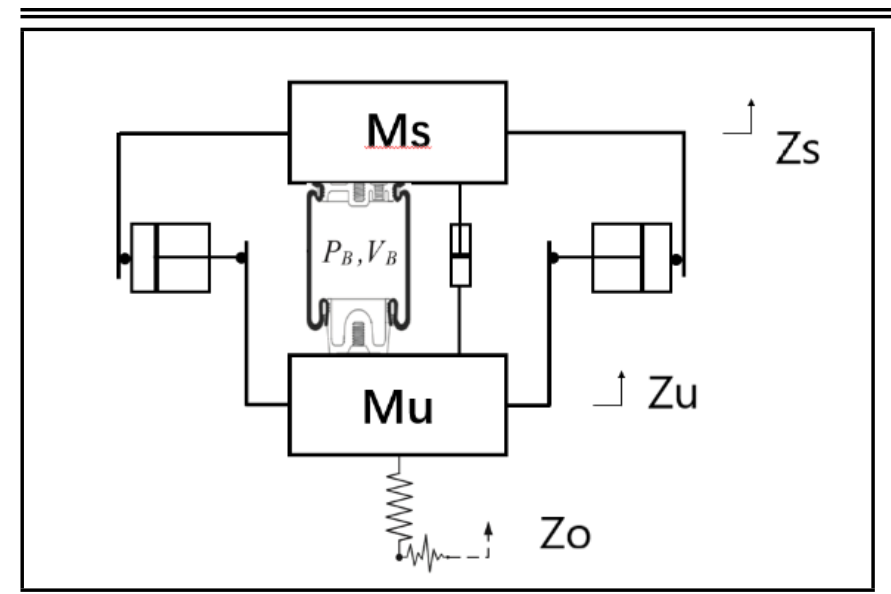

Figure 1. The physical model of quasi-zero stiffness air suspension.

which will be useful for the utilization of a quasi-zero stiffness structure in the vehicle suspension.

The remainder of this paper is organized as follows. In Section 2, the mathematical models of quasi-zero stiffness air suspension and interconnected quasi-zero stiffness air suspension are established. In Section 3, the nonlinear characteristics of the mathematical models are analyzed, and the random input of road surface is designed. In Section 4, the vibration isolation characteristics are analyzed through simulation and experimentation according to the equilibrium stability constraint and the attraction domain constraint. In Section 5, the performance changes of interconnected quasi-zero stiffness air springs and quasi-zero stiffness air springs compared with air springs are summarized and conclusions are drawn.

\section{QUASI-ZERO STIFFNESS SUSPENSION PROPOSED}

In this section, the mathematical model of quasi-zero stiffness air suspension will be built and divided into two states: connected and disconnected. Additionally, various parameter values are provided. Next, the spring force and stiffness curve of the quasi-zero stiffness system will be obtained via simulation analysis.

The quasi-zero stiffness air suspension, as shown in Fig. 1, consists of an air spring, two pairs of air actuators (i.e., four $90^{\circ}$ spaced air actuators), a damper, a sprung mass and an unsprang mass. The four actuators are placed on each side of the sprung mass $M_{s}$, which is located within a classical one degree of freedom system formed by such sprung mass $M_{s}$ and a pneumatic spring. The air spring and air actuators are connected through pipelines with solenoid valves to control the connected state. Since the stiffness of the quasi-zero stiffness air suspension system is determined by the vertical resultant force of the actuators and the air spring, the performance of the system is related to the structural and functional parameters of the air spring and the air actuator.

\subsection{Air Spring}

The air spring can keep the natural frequency basically unchanged under different loads and allow the actuator to remain horizontal in its initial position under any load at the same time which is suitable for overcoming the sensitivity of the quasizero stiffness mechanism to loads. In this section, the $644 \mathrm{~N}$ air spring from the Contitech Company is used. The spring force consists of two parts. The first part depends on the change of spring pressure, and the second part comes from the structural damping force.

The spring force $F_{s}$ is shown in Eq. (1): ${ }^{16}$

$$
F_{s}=\left[P_{s 0}\left(\frac{V_{0}}{V_{s}}\right)^{n_{s}}-P_{a}\right] A_{s}-\frac{\delta_{s}}{2 \pi f}\left(\dot{z}_{s}-\dot{z}_{u}\right)
$$

where $f$ is the excitation frequency, $V_{0}$ is the initial volume of the air spring, $V_{s}$ is the measured volume of the air spring, $P_{s 0}$ is the initial pressure of the air spring, $P_{a}$ is the atmospheric pressure, $A_{s}$ is the effective area of air spring, $n_{s}$ is the gas polytropic index, and $\delta_{s}$ is the structural damping coefficient.

\subsection{Quasi-Zero Stiffness Suspension in the Non-Interconnected State}

The mathematical expression of suspension in the noninterconnected state is a second-order differential equation that includes the sprung mass ( $1 / 4$ body mass) and the unsprung mass. Its schematic diagram is shown in Fig. 1. The equivalent stiffness of the tire is also considered, while tire damping is ignored.

The specific expression is as follows:

$$
\begin{gathered}
M_{s} \ddot{z}_{s}=\left[P_{s 0}\left(\frac{V_{0}}{V_{s}}\right)^{n_{s}}-P_{a}\right] A_{s}-\frac{\delta_{s}}{2 \pi f}\left(\dot{z}_{s}-\dot{z}_{u}\right)+ \\
C_{s}\left(\dot{z}_{u}-\dot{z}_{s}\right)-M_{s} g+4 F_{P L A} \frac{z_{s}-z_{u}}{\sqrt{\left(z_{s}-z_{u}\right)^{2}+l_{0}^{2}}} \\
M_{u} \ddot{z}_{u}=K_{t}\left(z_{0}-z_{u}\right)-\left[P_{s 0}\left(\frac{V_{0}}{V_{s}}\right)^{n_{s}}-P_{a}\right] A_{s}+ \\
\frac{\delta_{s}}{2 \pi f}\left(\dot{z}_{s}-\dot{z}_{u}\right)-C_{s}\left(\dot{z}_{u}-\dot{z}_{s}\right)- \\
4 F_{P L A} \frac{z_{s}-z_{u}}{\sqrt{\left(z_{s}-z_{u}\right)^{2}+l_{0}^{2}}} ; \\
F_{P L A}=\left[P_{s 0}\left(\frac{V_{a 0}}{V_{a s}}\right)^{n_{s}}-P_{a}\right] A_{e} \\
M_{s} g=\left[P_{s 0}-P_{a}\right] A_{s}
\end{gathered}
$$

where $l_{0}$ is the initial distance between two connection points, $C_{s}$ is the suspension damping, $M_{s}$ is the sprung mass, $M_{u}$ is the unsprung mass, $V_{a 0}$ is the initial volume of the actuator, $V_{a s}$ is the actual volume of the actuator, and $A_{e}$ is the effective area of the actuator.

\subsection{Quasi-Zero Stiffness Suspension in the Interconnected State}

In order to introduce the interconnection effect into the model, a new interconnected quasi-zero stiffness air spring model is introduced. Figure 2 illustrates the connected quasizero stiffness air spring and the simplified diagram. In this 


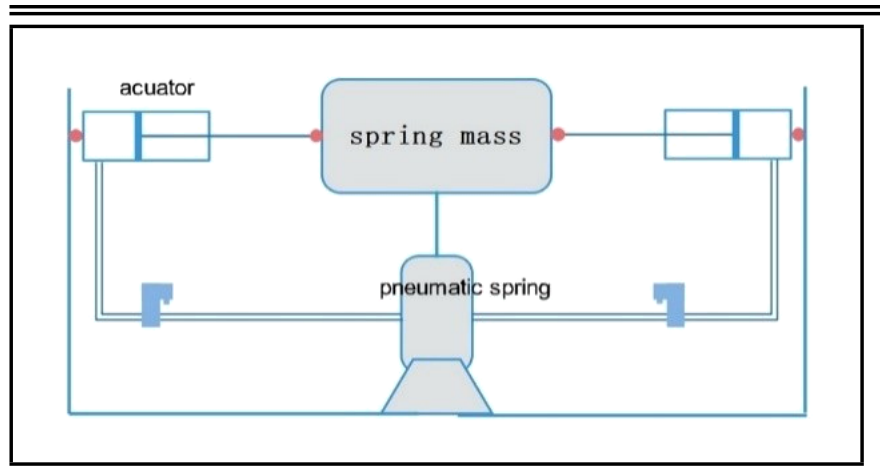

Figure 2. Interconnected quasi-zero stiffness air spring and the simplified diagram.

mechanism the actuators are placed opposite each other, in static conditions, they remain in a perpendicular direction to mass relative displacement. The end of the actuator rod is connected via a ball joint to the mass and another end is connected in the same way to the vibrating frame. The actuators and the pneumatic spring are connected via a pipeline controlled by a solenoid valve.

The expression of the interconnected 2-DOF suspension is as follows:

$$
\begin{aligned}
M_{s} \ddot{z}_{s}= & {\left[P_{s}-P_{a}\right] A_{s}-\frac{\delta_{s}}{2 \pi f}\left(\dot{z}_{s}-\dot{z}_{u}\right)+} \\
& C_{s}\left(\dot{z}_{u}-\dot{z}_{s}\right)-M_{s} g+4 F_{P L A} \frac{z_{s}-z_{u}}{\sqrt{\left(z_{s}-z_{u}\right)^{2}+l_{0}^{2}}}
\end{aligned}
$$

$$
\begin{gathered}
M_{u} \ddot{z}_{u}=K_{t}\left(z_{0}-z_{u}\right)-\left[P_{s}-P_{a}\right] A_{s}+ \\
\frac{\delta_{s}}{2 \pi f}\left(\dot{z}_{s}-\dot{z}_{u}\right)-C_{s}\left(\dot{z}_{u}-\dot{z}_{s}\right)- \\
4 F_{P L A} \frac{z_{s}-z_{u}}{\sqrt{\left(z_{s}-z_{u}\right)^{2}+l_{0}^{2}}}-M_{u} g \\
F_{P L A}=\left[P_{p}-P_{a}\right] A_{e} .
\end{gathered}
$$

By the dynamic adiabatic equation,

$$
\begin{gathered}
P_{s}=P_{s 0}\left(\frac{V_{10}}{m_{10}}\right)^{n_{s}}\left(\frac{m_{1}}{V_{1}}\right)^{n_{s}} ; \\
P_{p}=P_{s 0}\left(\frac{V_{20}}{m_{20}}\right)^{n_{s}}\left(\frac{m-m_{1}}{V_{2}}\right)^{n_{s}} .
\end{gathered}
$$

Also,

$$
\begin{gathered}
m_{1}=m_{10}+\int_{0}^{t} \dot{m}_{1} d t ; \\
V_{1}=V_{10}+A_{s}\left(z_{s}-z_{u}\right) ; \\
V_{2}=V_{20}+A_{e}\left(\sqrt{\left(z_{s}-z_{u}\right)^{2}+l_{0}^{2}}-l_{0}\right) ;
\end{gathered}
$$

where $m_{10}$ is the initial air mass of the air spring, $V_{10}$ is the initial volume of the air spring, $m$ is the initial air mass of the system, and $V_{20}$ is the initial volume of the actuator. In accordance with the literature, the mass velocity can be obtained shown in Eq. (7): ${ }^{16,17}$

$$
\dot{m}_{1}= \begin{cases}A_{p} p_{u} \sqrt{\frac{1}{R T_{u}} \frac{2 n_{s}}{n_{s}-1}\left[\left(\frac{p_{d}}{p_{u}}\right)^{\frac{2}{n_{s}}}-\left(\frac{p_{d}}{p_{u}}\right)^{\frac{n_{s}+1}{n_{s}}}\right]}, & \frac{p_{d}}{p_{u}}>v_{c r} \\ A_{p} p_{u} \sqrt{\left(\frac{2}{n_{s}+1}\right)^{\frac{n_{s}+1}{n_{s}-1}} \frac{n_{s}}{R T_{u}},} & \frac{p_{d}}{p_{u}} \leq v_{c r}\end{cases}
$$

where $A_{p}$ is the effective area of the pipeline, $p_{u}$ is the upstream gas pressure, $p_{d}$ is the downstream gas pressure, $T_{u}$ is the upstream gas temperature, $v_{c r}$ is a critical pressure ratio of 0.5282 , and $R$ is the ideal gas constant.

Due to the lag in pipe connection,

$$
\dot{m}_{1}(L, t)= \begin{cases}0, & t<L / c \\ e^{-\frac{R_{t} R T}{2 p_{d}} \frac{L}{c}} \dot{m}_{1}\left(0, t-\frac{L}{c}\right), & t \geq L / c\end{cases}
$$

where $L$ is the length of connecting pipeline, $T$ is the temperature of the connection pipeline $K, R_{t}$ is the damping coefficient of pipeline, and $c$ is the speed of sound.

Again,

$$
R_{t}= \begin{cases}\frac{32 \mu}{D^{2}}, & R_{e}<2600 \\ 0.1582 \frac{\mu}{D^{2}} R_{e}, & R_{e} \geq 2600\end{cases}
$$

where $R_{e}$ is the Reynolds number, $\mu$ is the kinematic viscosity coefficient of air, and $D$ is the diameter of the circular pipe.

Finally,

$$
\begin{aligned}
u & =4.8 \times 10^{-8} \times(T-273.15)+1.71 \times 10^{-5} ; \\
R_{e} & =\frac{\rho D V}{\mu}
\end{aligned}
$$

where $T$ is the air temperature in the pipeline, $\rho$ is the air density in the pipeline, $D$ is the diameter of the round tube, and $V$ is the average velocity of air.

\section{SUSPENSION STABILITY AND ATTRACTION DOMAIN}

The interconnected and non-interconnected quasi-zero stiffness air suspension is essentially a class of nonlinear vibration differential equations. Qualitative and quantitative research on this class of nonlinear vibration differential equations, including equilibrium stability and equilibrium attraction domain, has practical significance for the stability and safety in the actual use of this kind of suspension. ${ }^{18}$

\subsection{Stability Analysis of the Quasi-Zero Stiffness Air Suspension in the Non- Interconnected State}

The system could be simplified as a single mass quasi-zero stiffness system, and according to Eq. (2), the simplified equation could be obtained.

$$
\begin{aligned}
M_{s} \ddot{z}_{s}= & {\left[P_{s 0}\left(\frac{z_{0}}{z_{s}+z_{0}}\right)^{n_{s}}-P_{s 0}\right] A_{s}-\left(C_{s}+\frac{\delta_{s}}{2 \pi f}\right) \dot{z}_{s}+} \\
& 4 F_{P L A} \frac{z_{s}}{\sqrt{z_{s}^{2}+l_{0}^{2}}}
\end{aligned}
$$




$$
F_{P L A}=\left[P_{s 0}\left(\frac{d_{0}}{\sqrt{z_{s}^{2}+l_{0}^{2}}-l_{0}+d_{0}}\right)^{n_{s}}-P_{a}\right] A_{e}
$$

Let us set $c=C_{s}+\frac{\delta_{s}}{2 \pi f}, m=M_{s}, x=z_{s}, y=\frac{d x}{d t}$,

$$
\left\{\begin{aligned}
y= & \dot{x}=f_{1}(x, y) \\
\dot{y}= & -\frac{c}{m} y+\left\{\left[P_{s 0}\left(\frac{z_{0}}{x+z_{0}}\right)^{n_{s}}-P_{s 0}\right] \frac{A_{s}}{x}+\right. \\
& \left.4\left[P_{s 0}\left(\frac{d_{0}}{\sqrt{x^{2}+l_{0}^{2}}-l_{0}+d_{0}}\right)^{n_{s}}-P_{a}\right] \frac{A_{e}}{\sqrt{x^{2}+l_{0}^{2}}}\right\} \frac{1}{m} * x= \\
& f_{2}(x, y) .
\end{aligned}\right.
$$

In the system, the velocity and acceleration are set to zero and $n_{s}$ is the gas polytropic index, which can be regarded as $n_{s}=1$ at the quasi-static state. If $x=\frac{x}{l_{0}}, A=\frac{A_{s}}{A_{e}}, p=$ $\frac{P_{s 0}}{P_{a}}, d=\frac{d_{0}}{l_{0}}, z=\frac{z_{0}}{l_{0}}$, the restoring force of the system is dimensionless,

$\tilde{F}_{1}=\left[p \frac{z}{x+z}-p\right] A+4\left[p \frac{d}{\sqrt{x^{2}+1}-1+d}-1\right] \frac{x}{\sqrt{x^{2}+1}}=0$.

Therefore, the variable scale equilibrium equation of the autonomous system is,

$$
\left\{\begin{array}{l}
y=\dot{x}=f_{1}(x, y)=0 \\
\dot{y}=-c y+\tilde{F}_{1}=f_{2}(x, y)=0
\end{array}\right.
$$

It is known that $(x, y)=(0,0)$ is a solution to this equation. To verify its stability, the derivative of $\tilde{F}_{1}$ at this point with respect to $x$ is solved,

$$
\frac{\partial\left(\tilde{F}_{1}\right)}{\partial x}=-\frac{p A}{z}+4 p-4
$$

So, the differential equation near $(x, y)=(0,0)$ can be expressed as,

$$
\left[\begin{array}{l}
\dot{x} \\
\dot{y}
\end{array}\right]=\left[\begin{array}{cc}
0 & 1 \\
-\frac{p A}{z}+4 p-4 & -c
\end{array}\right]\left[\begin{array}{l}
x \\
y
\end{array}\right]=\mathbf{A}_{1} \cdot\left[\begin{array}{l}
x \\
y
\end{array}\right] .
$$

The eigenvalues of $\mathbf{A}_{1}$ are calculated. On the basis of the Lyapunov indirect method, when $t=\frac{p A}{z}+4 p-4 \leq-\frac{c^{2}}{4}$, i.e., $\gamma_{1,2}=\frac{-c}{2} \mp \sqrt{-t-\frac{c^{2}}{4}} i$, the eigenvalues are both on the left side of the complex plane and the equilibrium point is stable. When $t>-\frac{c^{2}}{4}$, i.e., $\gamma_{1,2}=\frac{-c}{2} \mp \sqrt{t+\frac{c^{2}}{4}}$, the case needs to be discussed: when $\frac{-c^{2}}{4}<t \leq 0$, the equilibrium point is stable, otherwise, the equilibrium point is unstable. According to the above analysis, when the dimensionless initial pressure $p$ is $\leq 4$ in the case of $A=1.5$ and $z=0.5$, the equilibrium point $(0,0)$ is stable. The different initial pressures $p$ and the initial positions $d$ of the actuator piston are selected to study the stability and suspension performance. ${ }^{18,19}$

When $t \leq 0$, that is, $p \leq 4$, it can be seen from the above judgment that $(0,0)$ is a stable point. If $d=0.1$, there is only one equilibrium point $(x, y)=(0,0)$ and it is a stable equilibrium point. If $d=0.4$, there are three equilibrium points, which are node $(0,0)$, a saddle point and the third node from negative to positive respectively on the $x$-axis. ${ }^{20,21}$ According to the Centre Manifold Theory, the saddle point can lead to bifurcation of the system dynamics behaviour, which directly affects the attraction domain of the stable point $(0,0)$, namely the stable amplitude of the suspension system.

Therefore, the study of the attraction domain at the equilibrium point $(x, y)=(0,0)$ is particularly important for the stability of the actual suspension system. The domain of attraction is shown as follows:

$$
D_{A}=\left\{x \in D \mid \varnothing(t ; x), \forall t \geq 0, \lim _{t \rightarrow \infty} \varnothing(t ; x)=0\right\} \text {. }
$$

In this paper, the attractive domain boundary is a type of special equilibrium point (saddle point), which is more suitable for combining the central manifold theorem and the cell-mapping method.

The array of equilibrium point is set as $E$, because the solution of $E$ is a hypersurface composed of the state variable $(x, y)$ and two parameters $(p, d)$. So, the diagram cannot be given directly in the three-dimensional space.

The velocity state variable $y$ is discarded and the set of solutions at the equilibrium position is gained.

$$
E=\{x \mid f(x, p, d)=0, x \in R, p \in[1,4), d \in[0,1)\} .
$$

$E$ in three-dimensional space is obtained by numerical method in Fig. 4. Taylor series expansion at $x=0,{ }^{23}$

$$
\begin{aligned}
& \tilde{F}_{1}=p A \sum_{n=0}^{\infty}(-n)^{n} z^{-n} x^{n}+ \\
& 4 x\left[\frac{p d}{1-d} \frac{\sum_{n=0}^{\infty} P_{n}(0) x^{n}}{1-m \sum_{n=0}^{\infty} P_{n}(0) x^{n}}-\frac{p d+1-d}{1-d} \sum_{n=0}^{\infty} P_{n}(0) x^{n}\right]
\end{aligned}
$$

where $P_{n}(x)=\frac{1}{z^{n+1} n !} \frac{d^{n}}{d x^{n}}\left(x^{2}-1\right)^{n}$ is the Legendre polynomial, and its few values are as follows: $P_{0}(0)=1, P_{1}(0)=0$, $P_{2}(0)=-\frac{1}{2}, P_{3}(0)=0, P_{4}(0)=\frac{3}{8}, P_{5}(0)=0, P_{6}(0)=$ $-\frac{5}{16}$. Based on bifurcation theory, ${ }^{24}$ when the velocity and acceleration are equal to zero, the original differential equation can be reduced to a polynomial of order 6 . At the same time, the bifurcation behaviour obtained is equivalent to the topology of the original equation. The following equation can therefore be obtained, ${ }^{22,24,26}$

$$
\begin{aligned}
& \left\{c_{1}(p, A, z, d)=-\frac{p A}{z}+4 p-4,\right. \\
& c_{2}(p, A, z, d)=\frac{2 p A}{z^{2}}, \\
& c_{3}(p, A, z, d)=-\frac{6 p A}{z^{3}}+12-\frac{12 p}{d}-12 p, \\
& c_{4}(p, A, z, d)=\frac{24 p A}{z^{4}} \\
& c_{5}(p, A, z, d)=-\frac{120 p A}{z^{5}}+180 p+\frac{180 p}{d}+\frac{120 p}{d^{2}}-180 \text {, } \\
& \left.c_{6}(p, A, z, d)=\frac{720 p A}{z^{6}}\right\}
\end{aligned}
$$




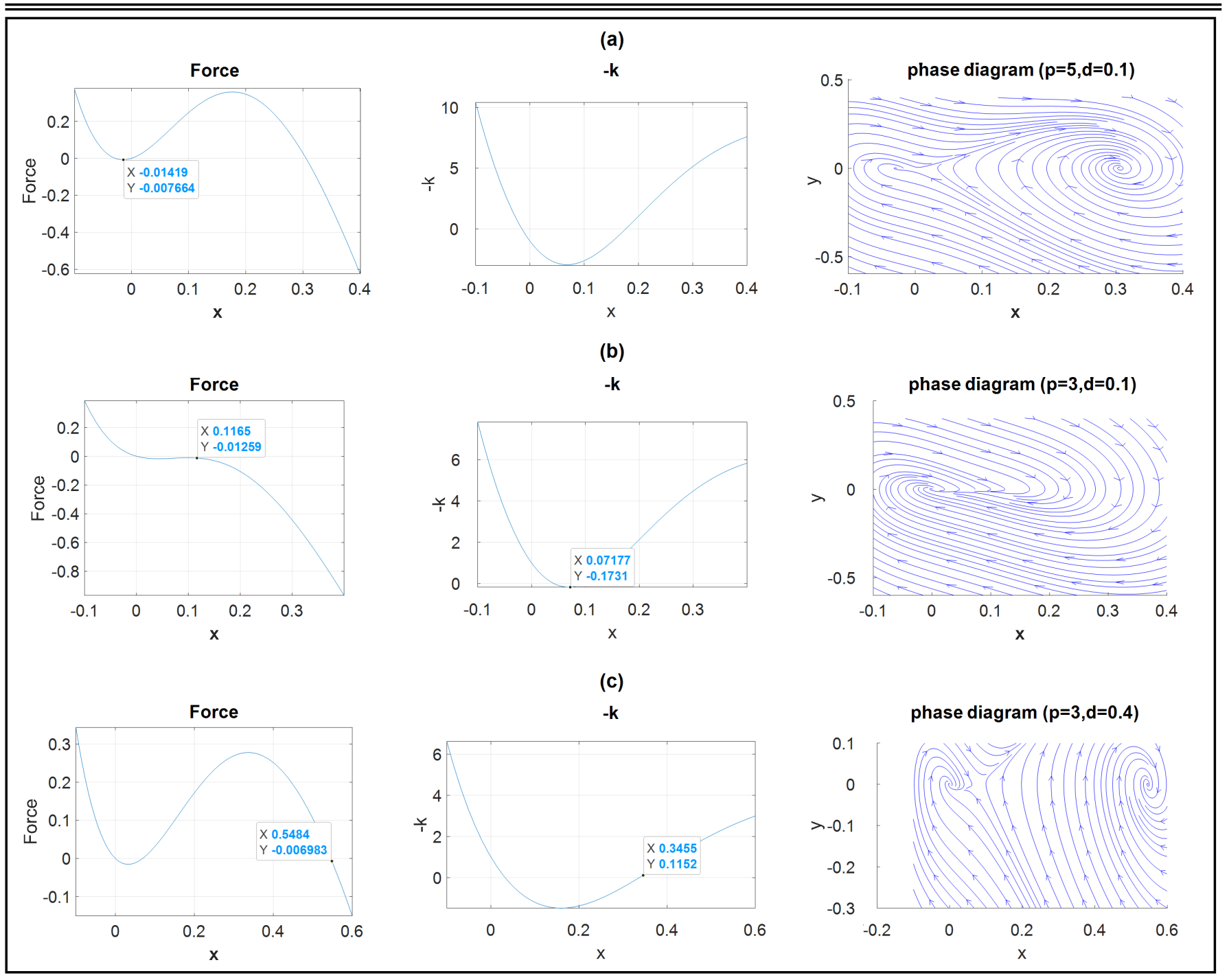

Figure 3. Phase diagram, restoring force and stiffness diagram of the non-interconnected suspension system for $A=1.5, z=0.5, c=1$, and different values of $(p, d)$ parameters: (a) $p=5, d=0.1$; (b) $p=3, d=0.1$; (c) $p=3, d=0.4$.

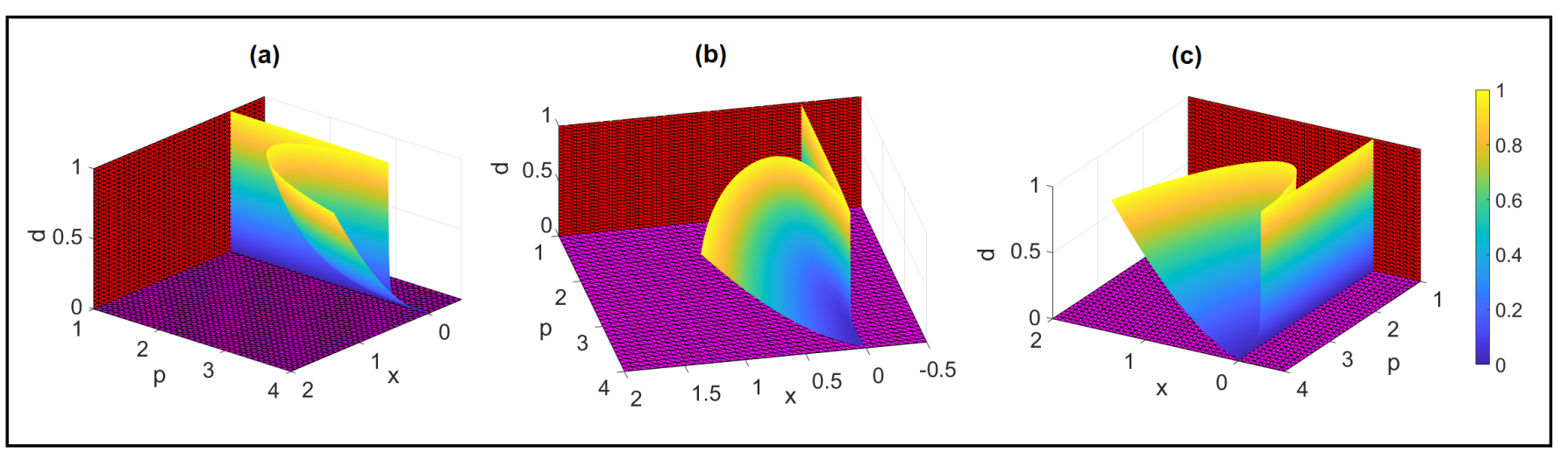

Figure 4. The set of equilibrium $x$ about the parameters $p \in[1,4)$ and $d \in[0,1)$ for $A=1.5, z=0.5$ : (a) left view; (b) top view; (c) right view.

$$
\tilde{F}_{1}=\sum_{n=1}^{6} c_{n} x^{n} .
$$

The basic idea of cell mapping is to discretize the system into many finitely sized units (cells) through a mapping relation, thus forming a discrete cell space and replacing the state space with the cell space. In this way, the transfer process of the continuous state of the system is correspondingly transformed into that of discrete cells, and the transfer relationship between cells represents the transfer relationship of the original system state. ${ }^{25,27}$

According to the principle of cell mapping algorithm, the system is discretized into

$$
\left\{\begin{array}{l}
x_{k+1}=y_{k} t+x_{k} ; \\
y_{k+1}=\left[\tilde{F}_{1}\left(x_{k}\right)-c y_{k}\right] t+y_{k} .
\end{array}\right.
$$

Partition primitive cell $Z=\left(Z_{1}, Z_{2}, \ldots, Z_{N}\right)$ gives the inequality, $\left(Z_{k}-1 / 2\right) h_{k} \leq\left(x_{k}, p_{k}, d_{k}\right)<\left(Z_{k}+1 / 2\right) h_{k}$, where 


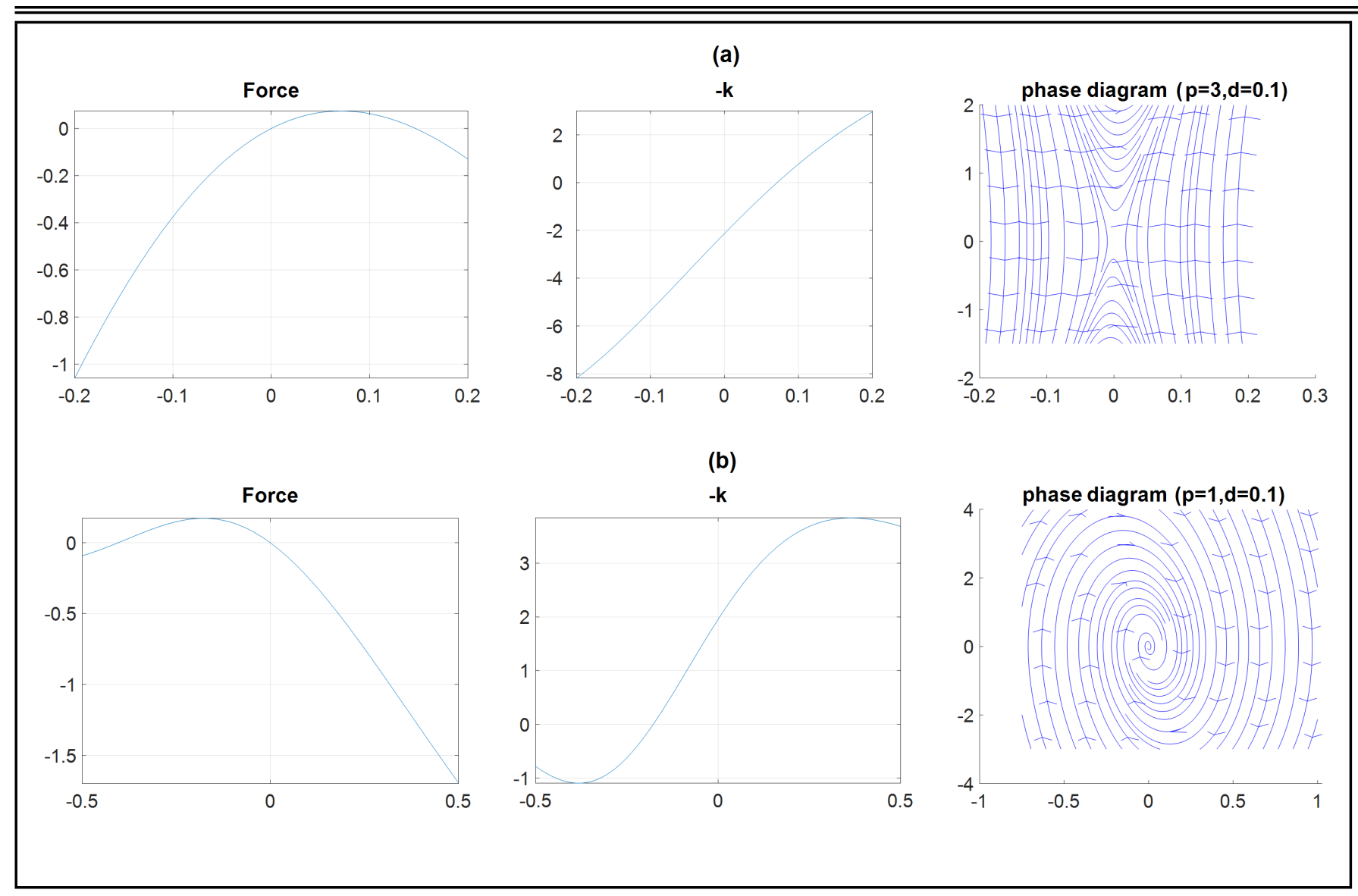

Figure 5. Phase diagram, restoring force and stiffness diagram of the suspension system for $A=1.5, z=0.5, c=1$, and different values of $(p, d)$ parameters: (a) $p=3, d=0.1 ;$ (b) $p=1, d=0.1$.

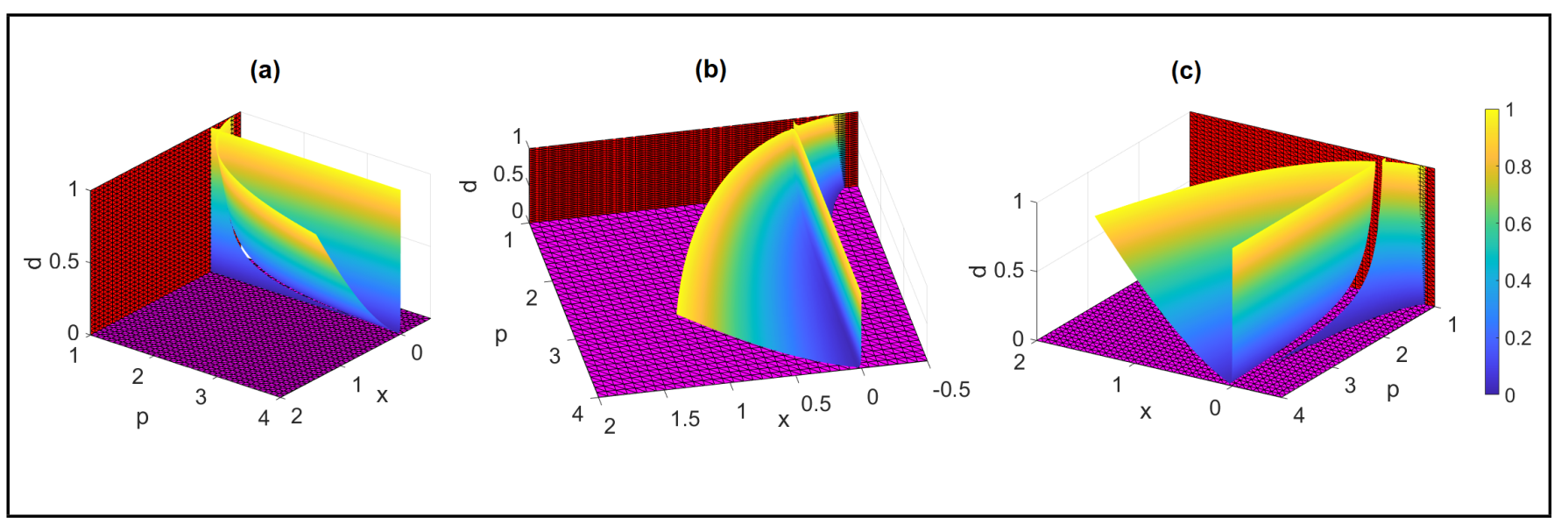

Figure 6. The set of equilibrium $x$ about the variables $p \in[1,4)$ and $d \in[0,1)$ for $A=1.5, z=0.5$ : (a) left view; (b) top view; (c) right view.

$h_{k}$ represents the distance between cells. The original cell was taken as the initial condition, and the following judgment criteria were designed. After $r$-step iterations, the actual dynamic behaviour was judged according to the following criteria:

A. The regression of the initial cell indicates that the result of the system evolution process is the periodic cell, indicating the stable periodic oscillation of the suspension.

B. The final cell belongs to the r-step attraction domain of the trap cell (cell outside the feasible domain), which indicates suspension instability.

C. The result is mapped to the primitive cell corresponding to the stable point $(0,0)$, indicating that the suspension is stable at the required stable point.

D. The cell needs $r$ steps to map the primitive cell and exclude the $(0,0)$ cell, so the suspension balances at a non$(0,0)$ point in the feasible region.

\subsection{Stability Analysis of the Quasi-Zero Stiffness Air Suspension in the Interconnected State}

Under the condition of interconnection, $n_{s}$ is the gas polytropic index, which could be regarded as $n_{s}=1$ at the quasistatic state. If $x=\frac{x}{l_{0}}, A=\frac{A_{s}}{A_{e}}, p=\frac{P_{s 0}}{P_{a}}, d=\frac{d_{0}}{l_{0}}, z=\frac{z_{0}}{l_{0}}$, the 
restoring force of the system is dimensionless,

$$
\begin{aligned}
\tilde{F}_{2}= & p A\left[\frac{A z+4 d}{A(x+z)+4\left[d-1+\sqrt{1+x^{2}}\right]}-1\right]+ \\
& 4\left[p \frac{A z+4 d}{A(x+z)+4\left[d-1+\sqrt{1+x^{2}}\right]}-1\right] \frac{x}{\sqrt{x^{2}+1}} .
\end{aligned}
$$

The $\tilde{F}_{2}$ at equilibrium $(0,0)$ to the partial derivative of $x$ is solved,

$$
\frac{\partial\left(\tilde{F}_{2}\right)}{\partial x}=4 p-\frac{A^{2} p}{4 d+A z}-4 .
$$

So, the suspension system near $(x, y)=(0,0)$ can be expressed as,

$$
\left[\begin{array}{c}
\dot{x} \\
\dot{y}
\end{array}\right]=\left[\begin{array}{cc}
0 & 1 \\
4 p-\frac{A^{2} p}{4 d+A z}-4 & -c
\end{array}\right]\left[\begin{array}{l}
x \\
y
\end{array}\right]=\mathbf{A}_{2} \cdot\left[\begin{array}{l}
x \\
y
\end{array}\right] .
$$

From the above equation, it can be seen that the suspension system's equilibrium state at $(0,0)$ in a connected state is not only related to the structural parameters $A$ and $z$, but also related to variable parameters $p$ and $d$. The eigenvalue of the matrix $\mathbf{A}_{2}$ is found to determine the stability by the Lyapunov indirect method. When $t=4 p-\frac{A^{2} p}{4 d+A z}-4 \leq-\frac{c^{2}}{4}$, i.e., $\gamma_{1,2}=-\frac{c}{2} \mp \sqrt{-t-\frac{c^{2}}{4}} i$, the eigen values are both on the left of the complex plane, so the equilibrium point is stable; when $t>-\frac{c^{2}}{4}, \gamma_{1,2}=-\frac{c}{2} \mp \sqrt{t+\frac{c^{2}}{4}}$, the results can be discussed as follows: when $-\frac{c^{2}}{4}<t \leq 0$, the equilibrium point is stable, and when $t>0$, the equilibrium point is unstable. According to the above analysis, when $p$ is $\leq 1+\frac{9}{64 d+3}$ in the case of $A=1.5$ and $z=0.5$, the system is stable at $(0,0)$. Therefore, it can be proven that the initial pressure $p$ of the interconnection and the initial position $d$ of the negative stiffness actuator piston jointly determines the stability of the system at the equilibrium point.

Comparing Fig. 5 and Fig. 3, it is shown that under the same initial conditions ( $p=3, d=0.1)$, the stable node evolves into a saddle point, i.e., the local stability evolves into instability when the quasi-zero stiffness suspension system transfers from a non-interconnected to an interconnected state. So, the research on the variation of the domain of attraction has a practical significance for air suspension control and parameter optimization.

The three-dimensional representation of its solution space is also obtained by a numerical method in Fig. 6. Taylor series expansion at $x=0$,

$$
\begin{aligned}
& \tilde{F}_{2}=p A\left[\frac{(A z+4 d) \sum_{n=0}^{\infty} P_{n}(0) x^{n}}{(A x+A z+4 d-4) \sum_{n=0}^{\infty} P_{n}(0) x^{n}+4}-1\right]+ \\
& 4 x \sum_{n=0}^{\infty} P_{n}(0) x^{n}\left[p \frac{(A z+4 d) \sum_{n=0}^{\infty} P_{n}(0) x^{n}}{(A x+A z+4 d-4) \sum_{n=0}^{\infty} P_{n}(0) x^{n}+4}-1\right] .
\end{aligned}
$$

(a)

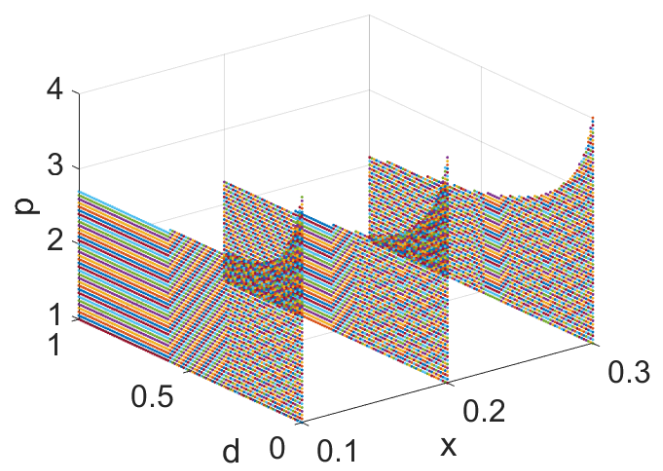

(b)

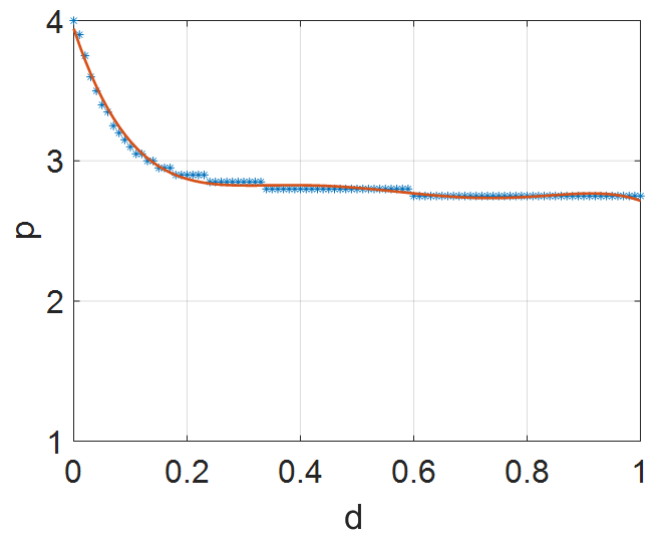

Figure 7. For $A=1.5, z=0.5$ : (a) the set of variable parameter $p$ and $d$ under different attraction domain $x$ for the non-interconnected quasi-zero stiffness air suspension; (b) the feasible domain of $p$ and $d$ when $x \in(-0.1,0.1)$.

(a)

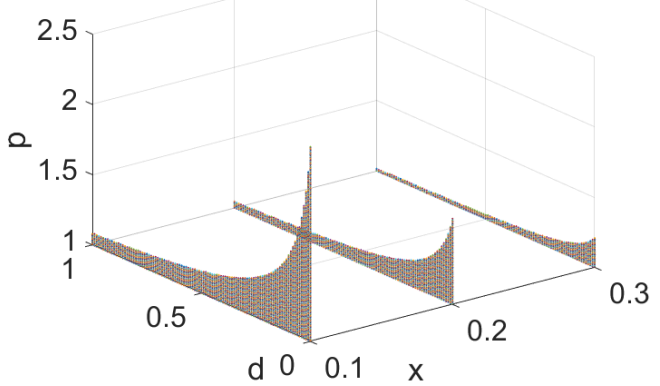

(b)

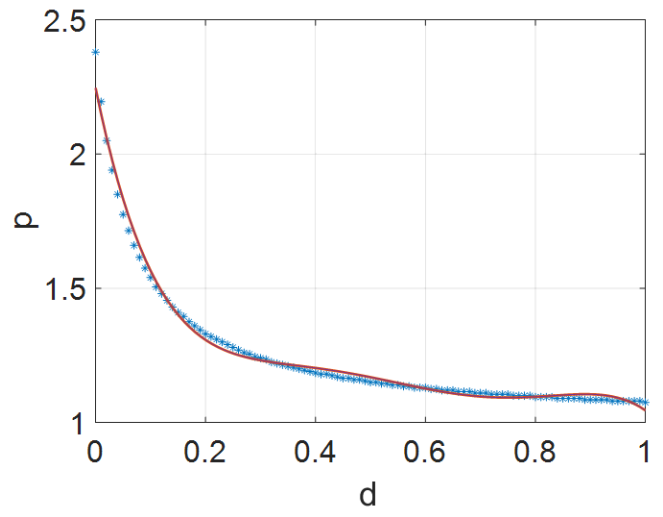

Figure 8. For $A=1.5, z=0.5$ : (a) the set of variable parameter $p$ and $d$ under different attraction domain $x$ for the interconnected suspension; (b) the feasible domain of $p$ and $d$ when $x \in(-0.1,0.1)$. 


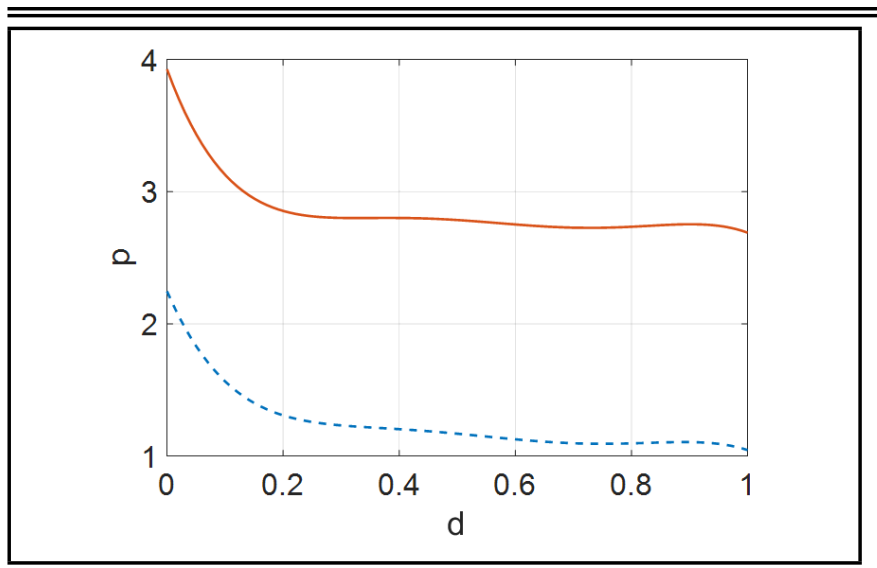

Figure 9. For $A=1.5, z=0.5$, the feasible domain of parameter $p$ and $d$ when attraction domain $x \in(-0.1,0.1)$ is the area bounded by the blue dotted line and the coordinate axis.

According to the bifurcation theory, when the velocity and acceleration of the original differential equation is equal to zero, it can be reduced to a polynomial of order 6 , which guarantees that the higher order terms of more than 6 times can be omitted theoretically, and the bifurcation behaviour obtained is equivalent to the original equation topologically. The following topological equivalent formula is obtained:

$$
\begin{aligned}
& \left\{c_{1}(p, A, z, d)=-\frac{p A^{2}}{4 d+A z}+4 p-4,\right. \\
& c_{2}(p, A, z, d)=\frac{2 p A^{3}}{(4 d+A z)^{2}}-\frac{12 p A}{4 d+A z}, \\
& c_{3}(p, A, z, d)=\frac{48 p A^{2}}{(4 d+A z)^{2}}-\frac{48 p}{4 d+A z}-12 p- \\
& \frac{6 p A^{4}}{(4 d+A z)^{3}}+12 \text {, } \\
& c_{4}(p, A, z, d)=\frac{24 p A^{5}}{(4 d+A z)^{4}}-\frac{240 p A^{3}}{(4 d+A z)^{3}}+\frac{60 A p}{4 d+A z}+ \\
& \frac{480 p A}{(4 d+A z)^{2}} \text {, } \\
& c_{5}(p, A, z, d)=180 p-180-\frac{120 p A^{6}}{(4 d+A z)^{5}}+ \\
& \frac{1440 p A^{4}}{(4 d+A z)^{4}}-\frac{4320 p A^{2}}{(4 d+A z)^{3}}- \\
& \frac{360 p A^{2}}{(4 d+A z)^{2}}+\frac{1920 p}{(4 d+A z)^{2}}+\frac{720 p}{4 d+A z}, \\
& c_{6}(p, A, z, d)=\frac{720 p A^{7}}{(4 d+A z)^{6}}+\frac{10080 p A^{5}}{(4 d+A z)^{5}}+ \\
& \frac{40320 p A^{3}}{(4 d+A z)^{4}}+\frac{2520 p A^{3}}{(4 d+A z)^{3}}- \\
& \left.\frac{40320 p A}{(4 d+A z)^{3}}-\frac{10080 A p}{(4 d+A z)^{2}}-\frac{1260 A p}{4 d+A z}\right\} \text {; }
\end{aligned}
$$

$$
\tilde{F}_{2}=\sum_{n=1}^{6} c_{n} x^{n} .
$$

(a)

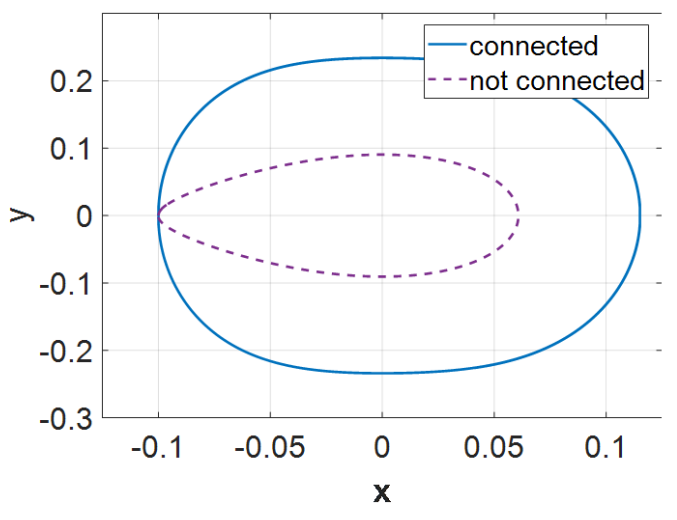

(b)

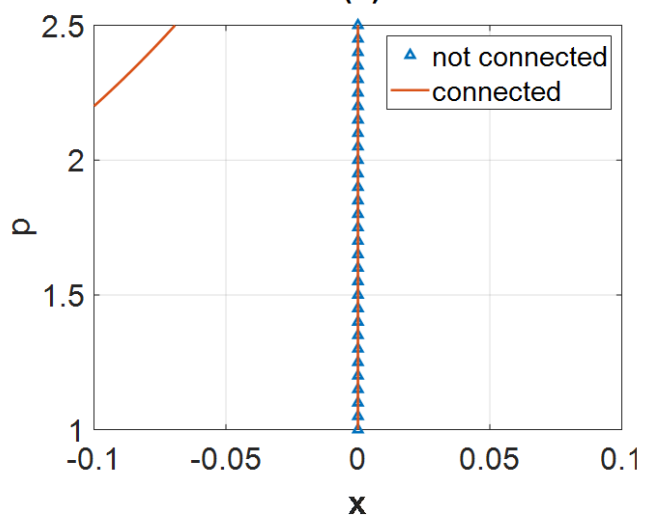

(c)

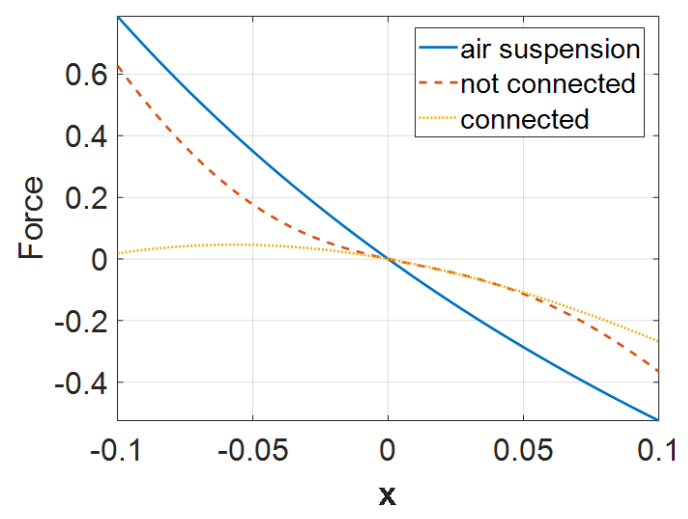

(d)

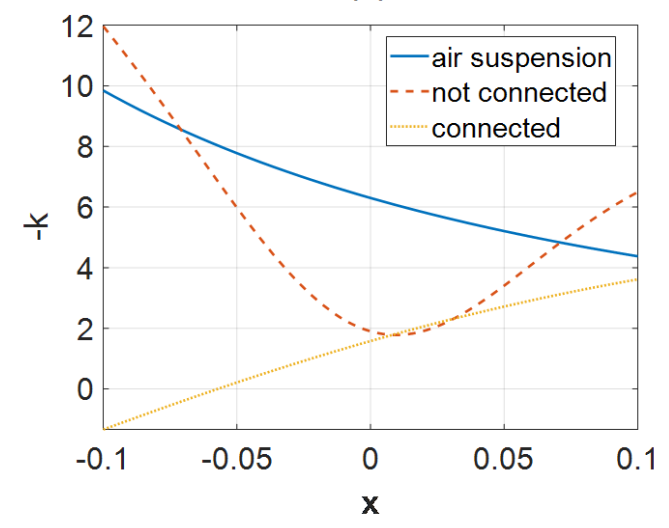

Figure 10. For $A=1.5, z=0.5, d=0.01, p=2.1$ : (a) attraction domain diagram, (b) bifurcation diagram, (c) recovery force diagram, (d) stiffness diagram. 
Table 1.

\begin{tabular}{|c|c|c|}
\hline & $\begin{array}{c}\text { The attraction } \\
\text { domain for } x\end{array}$ & $\begin{array}{c}\text { The stiffness } \\
\text { at stable point }\end{array}$ \\
\hline $\begin{array}{c}\text { The disconnected } \\
\text { quasi-zero suspension }\end{array}$ & 0.043 & 2 \\
\hline $\begin{array}{c}\text { The connected } \\
\text { quasi-zero suspension }\end{array}$ & $0.013(\downarrow 70 \%)$ & $1.8(\downarrow 10 \%)$ \\
\hline
\end{tabular}

\subsection{Analysis of Characteristics of Interconnected and Non-Interconnected Quasi-Zero Stiffness Air Suspension}

The suspension is expected to be stable at workspace $\mp 50 \mathrm{~mm}$ which is the common working space of suspension and is equivalent to dimensionless $\{x \mid-0.1<x<0.1\} \in$ $D_{A}\{x\}$, where $D_{A}\{x\}$ is the attracting domain. According to the general suspension parameters, the $A$ and $z$ is determined. The following Fig. 7 of the attraction domain is produced from cell mapping for the non-interconnected quasi-zero stiffness air suspension. The fitting curve of the boundary for the set $p$ and $d$ is as follows:

$$
\begin{aligned}
p= & -29.3880 d^{5}+85.9157 d^{4}-94.8521 d^{3}+49.1185 d^{2}- \\
& 12.0310 d+3.9000 .
\end{aligned}
$$

In the similar way, the feasible region of $p$ and $d$ for the interconnected suspension can be obtained, as shown in Fig. 8. The fitting curve of the boundary for the set $p$ and $d$ is as follows:

$$
\begin{aligned}
p= & -23.7156 d^{5}+68.9313 d^{4}-75.8102 d^{3}+39.4597 d^{2}- \\
& 10.0663 d+2.2000 .
\end{aligned}
$$

According to the above analysis, the stability of the quasizero stiffness air suspension in the switchable connected state with the same initial air pressure of the air spring and actuators at static equilibrium should meet the following constraints as shown in the Fig. 9.

$$
\left\{\begin{aligned}
p \leq & -29.3880 d^{5}+85.9157 d^{4}-94.8521 d^{3}+ \\
& 49.1185 d^{2}-12.0310 d+3.9000 \\
p \leq & -23.7156 d^{5}+68.9313 d^{4}-75.8102 d^{3}+ \\
& 39.4597 d^{2}-10.0663 d+2.2000 \\
p> & 1 .
\end{aligned}\right.
$$

When $d=0.01$, pressure $p=2.1$ can be found from the constraint conditions, $p \leq 2.1496$. The quasi-zero effect and the attraction domain of connected and disconnected equations can be obtained as follows in Fig. 10.

From the above analysis, it is shown that from the disconnected quasi-zero system to the connected quasi-zero system, the attraction region decreases, the stiffness changes are gentler, and the nonlinear characteristics are enhanced.

\subsection{Random Road Excitation}

Random roads can be determined in terms of a roughness coefficient $G_{q}$ which is defined in Table 2 for typical road classes according to ISO/TC108.

Urban road surface and high-speed road section are selected as the main simulation environments, which is sensitive for
Table 2.

\begin{tabular}{|c|c|c|c|c|}
\hline The road level & $\mathrm{A}$ & $\mathrm{B}$ & $\mathrm{C}$ & $\mathrm{D}$ \\
\hline$G_{q}\left(n_{0}\right)\left(* 10^{-6}\right)$ & 16 & 64 & 256 & 1024 \\
\hline
\end{tabular}

(a)

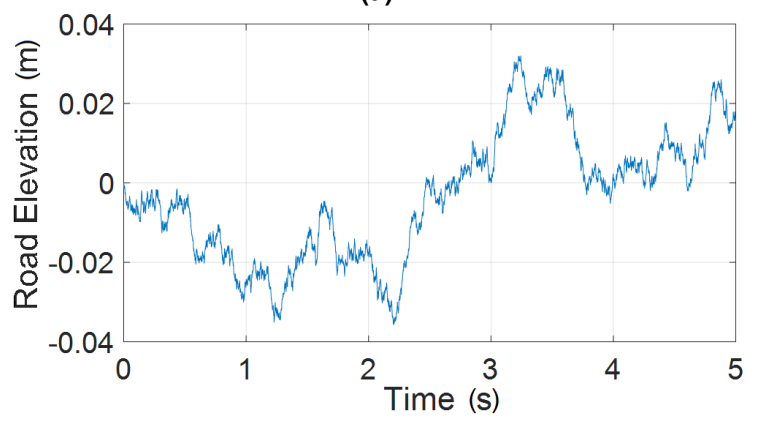

(b)

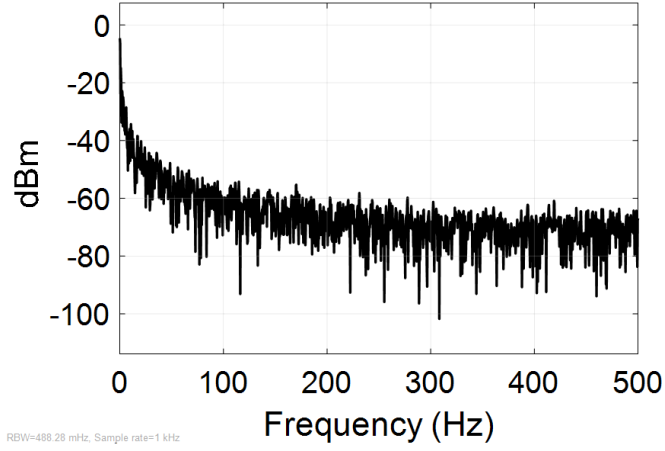

Figure 11. Time domain diagram and frequency domain diagram of road surface input.

comfort, so pavement roughness and driving speed are selected as a grade $C$ pavement of $20 \mathrm{~m} / \mathrm{s}(72 \mathrm{~km} / \mathrm{h})$. The pavement expression is shown in Eq. (31) and Fig. 11:

$$
\dot{Z}_{0}(t)+2 \pi f_{0} Z_{0}(t)=2 \pi n_{0} \sqrt{G_{q}\left(n_{0}\right) u(t)} w(t) ;
$$

where $n_{0}$ represents the spatial frequency of $0.1 \mathrm{~m}^{-1}$. The minimum boundary frequency of $f_{0}$ is $0.0628 \mathrm{~Hz} . u(t)$ is the speed in $\mathrm{m} / \mathrm{s} ; w(t)$ is white noise. ${ }^{28}$

\section{RESULTS}

Based on the Eq. (13), due to $\frac{\partial f_{1}}{x}+\frac{\partial f_{2}}{x}=-\frac{c}{m}$, the Bendixson Criterion determines that when $m c \neq 0$, there is no periodic closed orbit in the system, i.e. there is no stable periodic oscillation of the sprung mass in the above suspension system when damping exists. ${ }^{19}$

To verify the validity of the parameters selected in this paper, random road input was used to compare the smoothness and maneuverability of the disconnected quasi-zero stiffness air suspension and the connected quasi-zero stiffness air suspension with the passive air suspension. The initial parameters are shown in Table 3.

The simulation results of the air spring model are shown in Fig. 12. The stiffness varies from 43 to $100 \mathrm{kN} / \mathrm{m}$ when the suspension deflects from -40 to $50 \mathrm{~mm}$. The stiffness at equilibrium position is about $68150 \mathrm{~N} / \mathrm{m}$.

The power spectral density (PSD), time response of vehicle acceleration, suspension dynamic deflection and tire dynamic load were calculated. The simulation results are shown 


\begin{tabular}{||c|c|c||}
\hline \hline Table 3. Initial parameters. \\
\hline \multirow{3}{*}{ Suspension } & Sprung mass & $892.7 \mathrm{~kg}$ \\
parameters & Unspring mass & $415 \mathrm{~kg}$ \\
& Tire stiffness & $650 \mathrm{kN} / \mathrm{m}$ \\
& Damper damping & $9900 \mathrm{Ns} / \mathrm{m}$ \\
\hline \multirow{4}{*}{ Spring } & Radial effective area & $0.04 \mathrm{~m}^{2}$ \\
parameters & Absolute pressure & $0.32 \mathrm{MPa}$ \\
& Spring height & $250 \mathrm{~mm}$ \\
& Structural damping coefficient & $2000 \mathrm{Ns} / \mathrm{m}$ \\
& The volume & 131 \\
\hline \multirow{4}{*}{ Actuator } & Position of piston & $5 \mathrm{~mm}$ \\
parameters & The piston radius & $100 \mathrm{~mm}$ \\
& Absolute pressure & $0.32 \mathrm{MPa}$ \\
& Distance between two connection points & $500 \mathrm{~mm}$ \\
& Number of air actuators & $4 \mathrm{units}$ \\
\hline & Pipe diameter & $0.1 \mathrm{~m}$ \\
& The speed of sound & $334 \mathrm{~m} / \mathrm{s}$ \\
Others & The length of the pipe & $1 \mathrm{~m}$ \\
& Adiabatic gas index & 1.4 \\
& Atmospheric pressure & $0.1013 \mathrm{MPa}$ \\
& Air density & $1.23 \mathrm{~kg} / \mathrm{m}^{3}$ \\
& Road excitation frequency & $10 \mathrm{~Hz}$ \\
& Structural damping coefficient & $2000 \mathrm{~N} / \mathrm{m}$ \\
\hline
\end{tabular}

in Fig. 13. According to the selected parameters of stability analysis, $p=2.1$, i.e., the initial pressure was 2.1 atmospheres, and $d=0.01$, i.e., the initial position of the actuator piston was $5 \mathrm{~mm}$. The power spectral density was analyzed in the 5 -second time domain and $15 \mathrm{~Hz}$ frequency domain.

Combined with the analysis in Fig. 10, the dynamic stiffness of the disconnected quasi-zero stiffness air suspension near the equilibrium point is reduced by nearly $60 \%$ relative to the air spring, the root mean square (RMS) of the body acceleration $(0.3763)$ is reduced by about $16 \%$, and the dynamic load of the wheel (2.8983) is reduced by about $13 \%$, it also brings about a negative impact of approximately $56 \%$ increase in the dynamic deflection of the suspension (0.0078).

Compared with the disconnected quasi-zero stiffness air suspension, the stiffness of the connected quasi-zero stiffness air suspension is more gentle and smaller in the suspension travel, therefore, the suspension dynamic deflection (0.0079) relative to the air spring has increased nearly $58 \%$, but the body acceleration (0.3387) is optimized by nearly $24 \%$ and the wheel dynamic load (2.4662) is reduced by nearly $26 \%$.

Above all, the connected system was more superior to the disconnected system in terms of comfort and wheel dynamic load with about $10 \%$ lower body acceleration and $15 \%$ lower dynamic tire load which are specifically shown in the Fig. 14.

A single-channel experimental bench was built, and sampling time was selected as $5 e-6$ second to obtain sampling points with an interval of $0.2 \mathrm{~Hz}$. By calculating the ratio of the spring mass acceleration to the input acceleration, the transmissibility is expressed in the frequency domain as follows in the Fig. 15.

The simulation and experimental results show similar trends, and the error between them is less than $13 \%$. The resonance frequency of the interconnected quasi-zero stiffness suspension is approximately $1 \mathrm{~Hz}$, which is clearly lower than the resonance frequency of the air suspension (about $1.8 \mathrm{~Hz}$ ). The transmissibility of the natural frequency of the connected quasi-zero stiffness suspension (4.166) also decreased by about $58 \%$ compared with that of the air suspension (9.604).

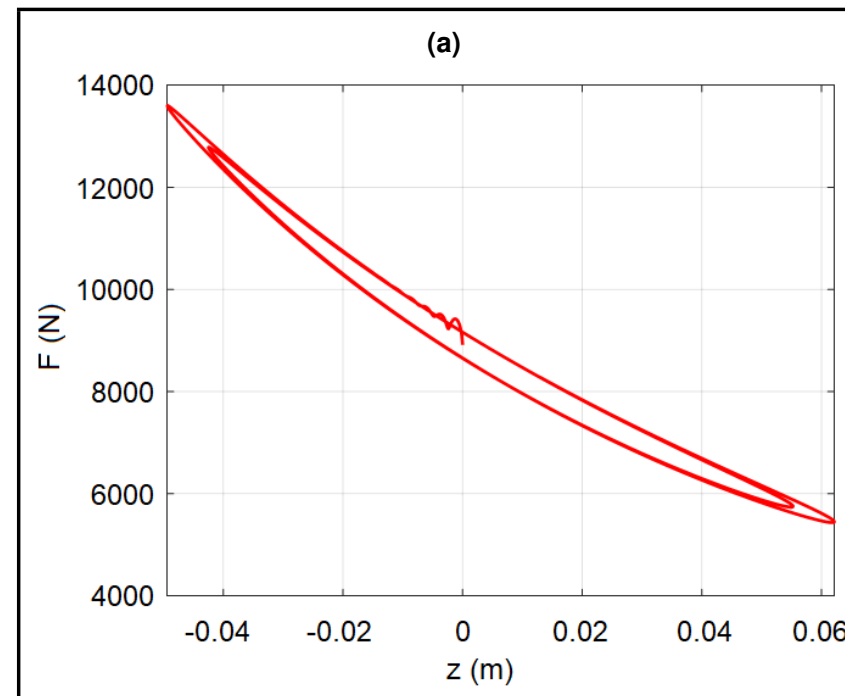

(b)

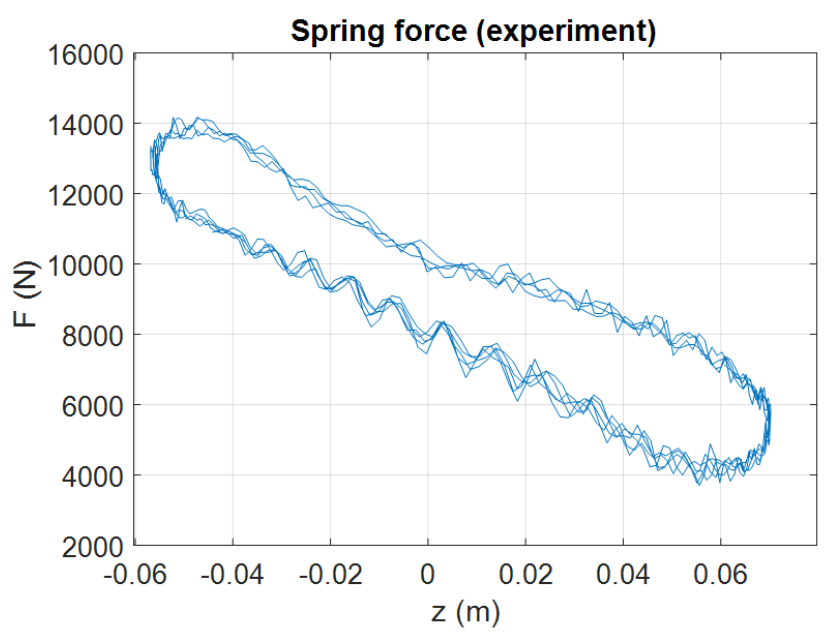

(c)

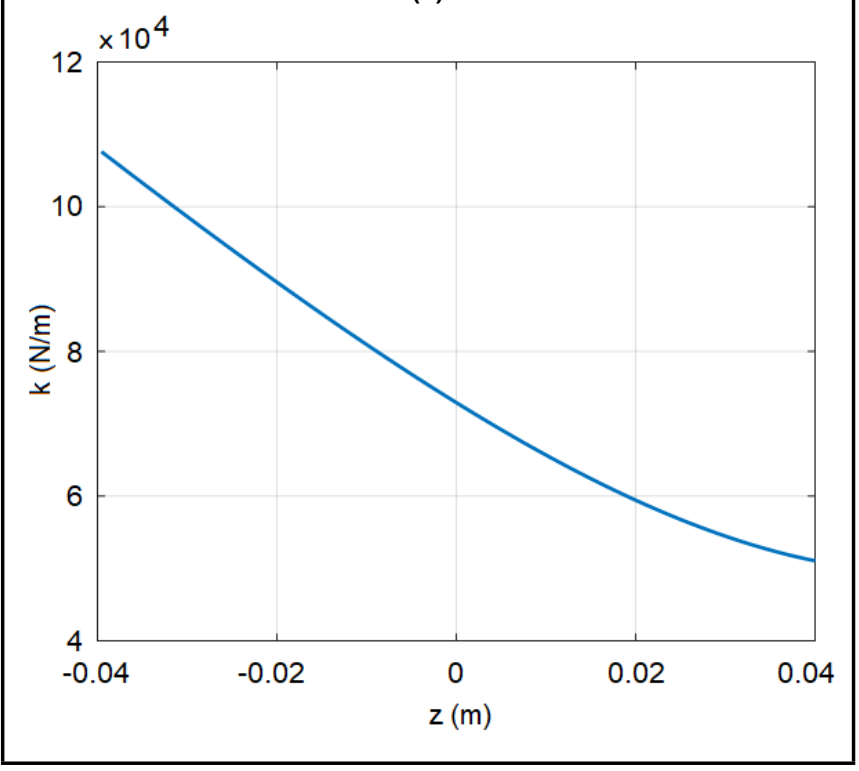

Figure 12. (a) Spring force (simulation), (b) spring force (experiment), (c) stiffness diagram for excitation frequency $10 \mathrm{~Hz}$, amplitude $-40 \sim$ $+50 \mathrm{~mm}$, initial absolute pressure $0.32 \mathrm{MPa}$. 


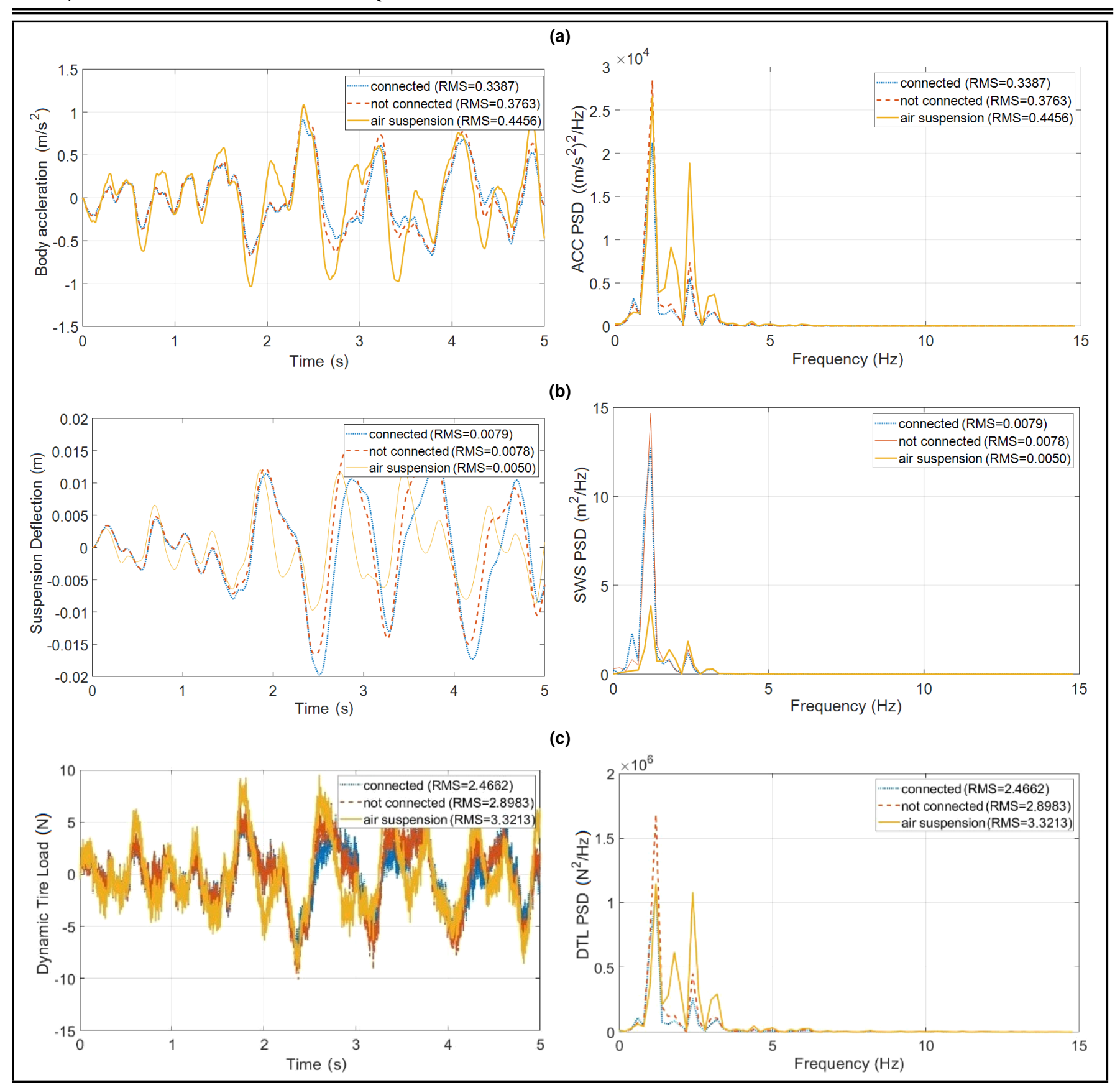

Figure 13. (a) Body acceleration (ACC), (b) suspension deflection (SWS), and (c) dynamic tire load (DTL) of air suspension, disconnected quasi-zero stiffness air spring and connected quasi-zero stiffness in time domain and frequency domain.

\section{CONCLUSIONS}

The main purpose of this paper was to study a quasi-zero stiffness air suspension with two states. The influence of the initial pressure and the initial position of the actuator piston on the stability of the equilibrium point and the attraction region of the equilibrium point was studied, under the premise of determining the air spring height, the effective area of the air spring and the distance between the two nodes of the actuator. The following conclusions were drawn.

1. In the disconnected quasi-zero stiffness air suspension, the stability of the equilibrium point was determined by initial pressure, relative area, and initial height, independent of the initial position of the piston. In the connected quasi-zero stiffness air suspension, the stability of the equilibrium point was determined by initial pressure, relative area, initial height and piston position.

2. The Bendixson Criterion determines that periodic vibration of the sprung mass will not occur to the studied system except for the lack of damping.

3. When the initial height $\mathrm{z}$ and effective area $A$ of the air spring was determined and the suspension maximum deflection was limited under $50 \mathrm{~mm}$ with the mode switching from the disconnected to the connected under stability constraints, the feasible region combined with initial pressure and initial piston position was reduced by about $60 \%$. The maximum workable initial pressure was also reduced from 4 atmospheres to 2.1 atmospheres.

4. Under the $C$ level road input, the suspension deflection of the connected quasi-zero stiffness suspension had nearly 


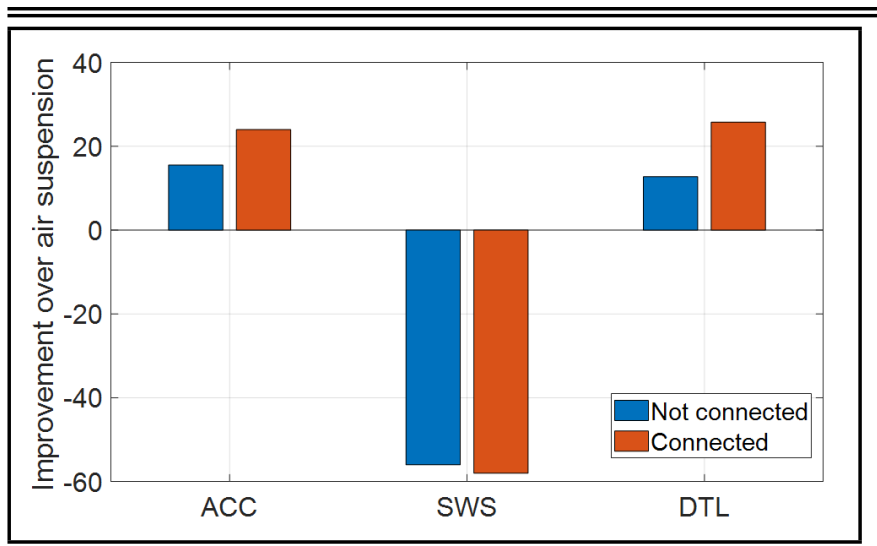

Figure 14. Comparison of the body acceleration, suspension deflection, and dynamic tire load between disconnected air spring and connected quasi-zero stiffness air spring.

similar degradation over the air suspension in comparison with that of the disconnected quasi-zero stiffness suspension, but the body acceleration and dynamic tire load of the connected system improved nearly twice as much as that of disconnected system.

In general, the connected quasi-zero stiffness air suspension reduces the parameter feasible region of $\mathrm{p}$ and $\mathrm{d}$ under the stability constraint and improved the attenuation effect of the system to the road acceleration and the dynamic load of the wheels.

\section{ACKNOWLEDGEMENTS}

The authors acknowledge the National Natural Science Foundation of China (Grant No.51875256) for financial support.

\section{REFERENCES}

1 Paddan, G. S. and Griffin, M. J. Evaluation of whole-body vibration in vehicles, Journal of Sound and Vibration, 253 (1), 195-213, (2002). https://dx.doi.org/10.1006/jsvi.2001.4256

2 Pawelczyk, M., Crocker, M. J. International Congress on Sound \& Vibration (ICSV16), Building Acoustics, 15 (4), 349-350, (2008). https://dx.doi.org/10.1260/135101008786939964

3 Moheyeldein, M. M., Abd-El-Tawwab, A. M., Abd ElGwwad, K. A., and Salem, M. M. M. An analytical study of the performance indices of air spring suspensions over the passive suspension, Beni-Suef University Journal of Basic and Applied Sciences, 7 (4), 525-534, (2018). https://dx.doi.org/10.1016/j.bjbas.2018.06.004

4 Virgin, L. N., Santillan, S. T., and Plaut, R. H. Vibration isolation using extreme geometric nonlinearity, Journal of Sound and Vibration, 315 (3), 721-731, (2008). https://dx.doi.org/10.1016/j.jsv.2007.12.025

5 Yin, Z., Khajepour, A., Cao, D., Ebrahimi, B., and Guo, K. Pneumatic suspension damping characterisation with equivalent damping ratio, International Journal of Heavy Vehicle Systems, 19 (3), 314-332, (2012). https://dx.doi.org/10.1504/ijhvs.2012.047919 (a)

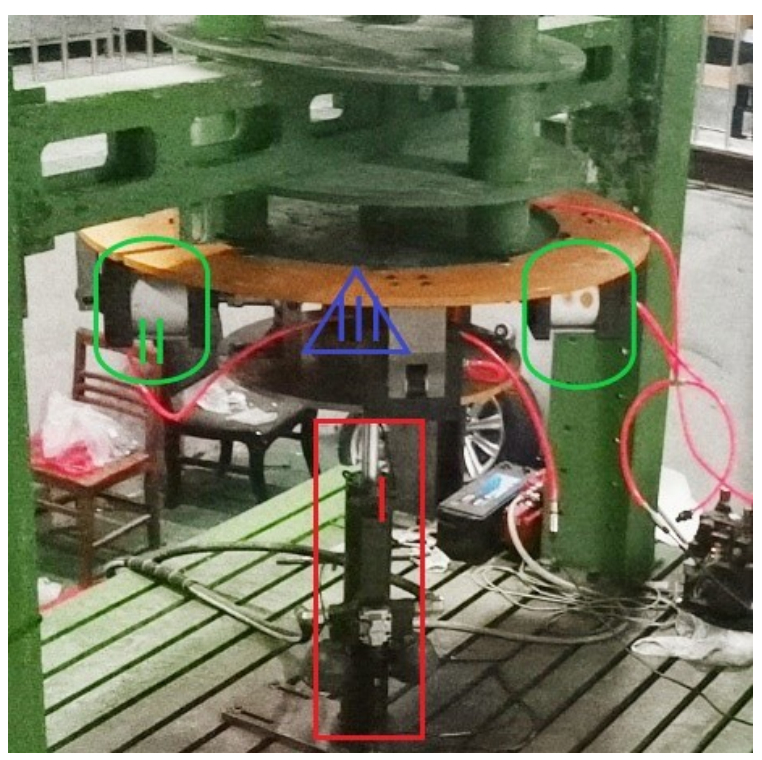

(b)

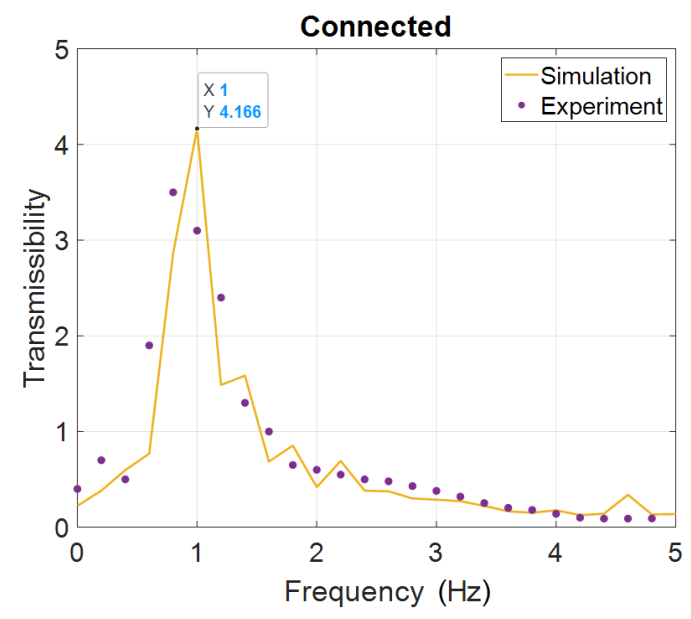

(c)

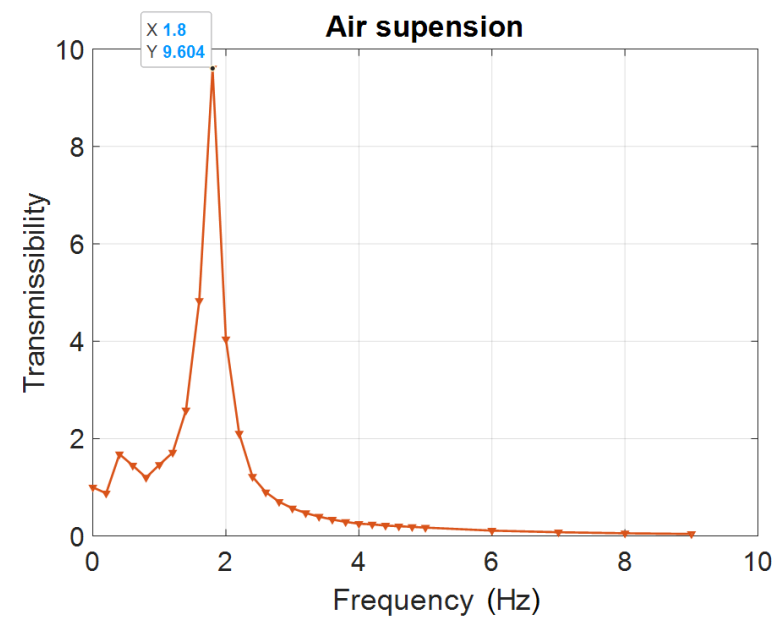

Figure 15. (a) Experimental bench (I: vibration platform, II: actuators, III: air spring); (b) simulation and experimental transmissibility of the connected quasi-zero stiffness air suspension, (peak value: $x=1, y=4.166$ ); (c) Experimental air suspension transmissibility (peak value: $x=1.8, y=9.604$ ). 
6 Zhou, J., Xiao, Q., Xu, D., Ouyang, H., and Li, Y. A novel quasi-zero-stiffness strut and its applications in six-degree-of-freedom vibration isolation platform, Journal of Sound and Vibration, 394, 59-74, (2017). https://dx.doi.org/10.1016/j.jsv.2017.01.021

7 Mizuno, T., Toumiya, T., and Takasaki, M. Vibration isolation system using negative stiffness, JSME International Journal Series C Mechanical Systems, Machine Elements and Manufacturing, 46 (3), 807-812, (2003). https://dx.doi.org/10.1299/jsmec.46.807

8 Xu, D., Yu, Q., Zhou, J., and Bishop, S. R. Theoretical and experimental analyses of a nonlinear magnetic vibration isolator with quasi-zero-stiffness characteristic, Journal of Sound and Vibration, 332 (14), 3377-3389, (2013). https://dx.doi.org/10.1016/j.jsv.2013.01.034

9 Hao, Z., Cao, Q., and Wiercigroch, M. Nonlinear dynamics of the quasi-zero-stiffness SD oscillator based upon the local and global bifurcation analyses, Nonlinear Dynamics, 87 (2), 987-1014, (2017). https://dx.doi.org/10.1007/s11071-016-3093-6

10 Gatti, G. A K-shaped spring configuration to boost elastic potential energy, Smart Materials and Structures, 28 (7), 077002, (2019). https://dx.doi.org/10.1088/1361$665 \mathrm{x} / \mathrm{ab} 1 \mathrm{ec} 8$

11 Ye, K., Ji, J. C., and Brown, T. Design of a quasi-zero stiffness isolation system for supporting different loads, Journal of Sound and Vibration, 471, 115198, (2020). https://dx.doi.org/10.1016/j.jsv.2020.115198

12 Gatti, G. Statics and dynamics of a nonlinear oscillator with quasi-zero stiffness behaviour for large deflections, Communications in Nonlinear Science and Numerical Simulation, 83, 105143, (2020). https://dx.doi.org/10.1016/j.cnsns.2019.105143

13 Tu, L., Ning, D., Sun, S., Li, W., Huang, H., Dong, M., and Du, H. A novel negative stiffness magnetic spring design for vehicle seat suspension system, Mechatronics, 68, 102370, (2020). https://dx.doi.org/10.1016/j.mechatronics.2020.102370

14 Ye, K., Ji, J. C., and Brown, T. A novel integrated quasi-zero stiffness vibration isolator for coupled translational and rotational vibrations, Mechanical Systems and Signal Processing, 149, 107340, (2021). https://dx.doi.org/10.1016/j.ymssp.2020.107340

15 Palomares, E., Nieto, A. J., Morales, A. L., Chicharro, J. M., and Pintado, P. Numerical and experimental analysis of a vibration isolator equipped with a negative stiffness system, Journal of Sound and Vibration, 414, 31-42, (2018). https://dx.doi.org/10.1016/j.jsv.2017.11.006

16 Jiang, H., Qian, K., Ju, L. Y., and Cui, Z. Simulation and experimental study on static and dynamic characteristics of laterally interconnected air suspension, Applied Mechanics and Materials, 577, 273-276, (2014). https://dx.doi.org/10.4028/www.scientific.net/amm.577.273
17 Li, Z., Ju, L., Jiang, H., and Xu, X. Imitated skyhook control of a vehicle laterally interconnected air suspension, International Journal of Vehicle Design, 74 (3), 204-230, (2017). https://dx.doi.org/10.1504/ijvd.2017.086430

18 Jerbi, H. M., Hamidi, F., Ben Aoun, S., Olteanu, S. C., and Popescu, D. Lyapunov-based methods for maximizing the domain of attraction, International Journal of Computers, Communications \& Control, 15 (5), (2020). https://dx.doi.org/10.15837/ijccc.2020.5.3898

19 Auersch, L. Emission of train-induced ground vibration-Prediction of axle-load spectra and its experimental verification, International Journal of Acoustics and Vibration, 22 (1), 74-83, (2017). https://dx.doi.org/10.20855/ijav.2017.22.1453

20 Nokhas, M., Kaki, M., and Aziz, S. A. Stability and existence of periodic solutions in non-linear differential equations, International Journal of Emerging Technologies in Learning, 3 (6), (2014). https://dx.doi.org/10.1007/978-94010-3323-7_15

21 Ueda, J. and Yoshikawa, T. Robust arm configuration of manipulator mounted on flexible base, Journal of the Robotics Society of Japan, 20 (5), 537-542, (2002). https://dx.doi.org/10.1109/tro.2004.829482

$22 \mathrm{Xu}, \mathrm{W}$. L. and Wu, R. H. Lyapunov's indirect method for stability analysis of fuzzy control system, Journal of Hunan University (Natural Sciences), 31 (3), 86-89, (2004). https://dx.doi.org/10.3321/j.issn:1000-2472.2004.03.020

23 Xi, X. C., Poo, A. N., and Hong, G. S. Taylor series expansion error compensation for a bi-axial CNC machine, Proc. of the 2008 IEEE International Conference on Systems, Man and Cybernetics, 1614-1619, (2008). https://dx.doi.org/10.1109/icsmc.2008.4811518

24 Sun, J. Q. and Hsu, C. S. The generalized cell mapping method in nonlinear random vibration based upon short-time gaussian approximation, Journal of Applied Mechanics, 57 (4), 1018-1025, (1990). https://dx.doi.org/10.1115/1.2897620

25 Sun, L. Simulation of pavement roughness and IRI based on power spectral density, Mathematics and Computers in Simulation, 61 (2), 77-88, (2003). https://dx.doi.org/10.1016/s0378-4754(01)00386-x

26 Qu, Z. Model Order Reduction Techniques with Applications in Finite Element Analysis, Springer London Ltd, England, UK, (2004).

27 Assaf, S. Finite element vibration analysis of damped composite sandwich beams, International Journal of Acoustics and Vibration, 16 (4), (2011). https://dx.doi.org/10.20855/ijav.2011.16.4293

28 Morton, P. Legendre polynomials and complex multiplication, I, Journal of Number Theory, 130 (8), 1718-1731, (2010). https://dx.doi.org/10.1016/j.jnt.2010.03.009 